\title{
Ubiquitous Access to Cultural Tourism Portals*
}

\author{
Franca Garzotto ${ }^{1}$ \\ Paolo Paolini ${ }^{1}$ \\ Marco Speroni ${ }^{1}$ \\ HOC-Hypermedia Open Center ${ }^{l}$ \\ Department of Electronics and Information \\ Politecnico di Milano, Italy
}

\author{
Birgit Pröll ${ }^{2}$ \\ Werner Retschitzegger ${ }^{3}$ \\ Wieland Schwinger ${ }^{4}$
IFS - Department of Information Systems ${ }^{3}$
TK - Department of Telecooperation ${ }^{4}$
Johannes Kepler University Linz, Austria \\ FAW - Institute of Applied Knowledge Processing ${ }^{2}$
}

\begin{abstract}
Web-based tourism information systems are more and more required to provide besides traditional tourism information about hotel facilities and infrastructure also cultural content comprising material heritage, performing art, folk tradition, handicraft or simply habits of everyday life. These cultural Web applications are required not to offer on-line brochures only, but rather to provide both, value and service. This paper focuses on two crucial aspects of cultural Web applications comprising quality of content and quality of access. As an example for achieving quality of content in terms of comprehensiveness and cross-national nature, the MEDINA portal is presented, allowing one-stop access to cultural information of fourteen Mediterranean countries. In order to provide quality of access, the notion of ubiquity is introduced, allowing to customize Web applications towards different kinds of contexts, thus supporting the cultural tourist with device-independent, time-aware, location-aware, and personalized services.
\end{abstract}

\section{Introduction}

The tourism industry can be seen as one of the first branches, where business has been done in an electronic way. Already in the 1960s, airline reservation systems have been employed which can be regarded as the forerunners of today's electronic commerce (EC) systems [17], [23]. Today, tourism information systems are one of the most important application areas for EC. The number of tourists who use Web-based tourism information systems for pre-trip planning jumped from 3.1 million in 1996 to 33.8 million in 1998 , a 1000 percent increase in two years [26]. It is furthermore predicted, that within the next 10 years, $30 \%$ of the whole tourism business will be done via Internet [24].
Over the last few years, a new form of so called "culture tourism" emerged. Cultural Web applications focus on the presentation of material and immaterial cultural heritage of an area including environmental and landscape attractions, historical, artistic, archaeological and folkloric aspects, traditions and rituals, handicraft objects and activities as well as gastronomy. The special properties of cultural content comprising heterogeneity, intangibility and perishability make them extremely information-intensive and thus especially suited for presenting them in an electronic way [29]. Not least due to that reason, an increasing proliferation of cultural Web applications can be observed, almost every cultural institution or initiative today wants to be on the Web, to promote itself and to disseminate its content and activities [18]. When developing cultural Web applications today, one has to keep in mind, however, that just to publish online brochures is by far not enough. Two crucial aspects can be identified which seem to be essential for the success of cultural Web applications, comprising quality of content and quality of access (cf. also [18]).

Quality of content means to provide comprehensive, potentially cross-national cultural information which is, at the same time, accurate, consistent and topical. One major building block technology which constitutes a prerequisite for achieving quality of content, while working against the proliferation of isolated, national or even regional cultural Web applications are portals [2], [9], [28]. A cultural Web portal can be used, first of all, as a central access point to a huge amount of potentially already available information at national or regional Web sites. Second, by combining the portal with an appropriate Extranet, a decentralised maintenance approach can be established, allowing to update the cultural content presented by the portal directly by each partner institution, thus facilitating topicality of information [19].

"This work is supported by the European Commission, under contract EUMEDIS 314 "MEDINA - MEDiterranean by INternet Access". 
To cope with the demand for quality of access, cultural Web applications should be made ubiquitous, thus allowing access with any media, at anytime, from anywhere [12], [13]. In this way, services can be offered which are device-independent, time-aware, locationaware, and personalized. This would assist the cultural tourist not only in the preparatory phase of a vacation but especially during the vacation itself, by inspiring his choice, e.g., via a PDA, where to go and how to organize an individual cultural tour.

Dealing with the two quality aspects of cultural Web applications described above, this paper is organized as follows: As an example for achieving quality of content, Section 2 gives an overview of the EU-supported MEDINA project which aims at establishing a cultural Web portal for fourteen Mediterranean countries. Section 3 introduces concepts in order to achieve ubiquitous access by proposing an architecture which allows to adapt a Web application towards a certain context. Section 4 applies these concepts to the MEDINA portal by discussing an exemplary scenario. Finally, Section 5 provides a brief summary of the paper.

\section{The MEDINA Project}

MEDINA (MEDiterranean by INternet Access), partially funded by the European Commission, includes seventeen partners from fourteen countries in the Mediterranean area: national and regional tourism offices, ministries of culture and tourism, universities and private corporations. It started in 2002 and will end in march 2005. In the following, the goals and the overall approach underlying the MEDINA project, the basic design decisions concerning the structuring of the cultural content and the technical architecture driving the development of the MEDINA portal are briefly outlined (for more details cf. [6]).

\subsection{Goals and Approach of MEDINA}

The current situation concerning existing cultural Web applications in the Mediterranean basin can be described as follows (cf. also [25]):

- Cultural heritage is advertised at small local Web sites only, there are no cross dimensions exploring cultural themes and linking them with tourism information.

- The diversities and identities of different regions combining environmental and landscape attractions, (im)material cultural heritage and local realities like handicraft are not properly represented.

- Cross-Mediterranean dimensions, like North-South linking aspects and common characteristics of the Mediterranean culture are not considered.

- There is no possibility to carry out "virtual cultural paths" or to get cultural-oriented itineraries which cross diverse themes and different geographic areas.
Based on this situation, MEDINA pursues the following objectives: First, a Mediterranean cultural Web portal ("MEDINA portal") should be established, promoting cultural tourism across the Mediterranean area, oriented towards both, end-users, directly organising their trips, and professional operators looking for specific information. Second, there should be a federation and adaptation of national Web sites, which organise information according to local points of view and focuses but share a common design in terms of structure and content topics. Third, the long-term operation and maintenance of the MEDINA portal and the national Web sites should be ensured after the end of the project.

The approach taken to realise these goals can be described as follows: In order to properly define the content structure of the MEDINA Portal and the national Web sites as well as to design a new matrix of "cultural pathways" within the Mediterranean area, goal-oriented requirements engineering is used [15]. To ensure interoperability and feasible enhancements of national Web sites, the design of the conceptual/logical schema of the MEDINA portal and of each national Web site is done by using the W2000 methodology which originated from a previously funded EU project [7]. To allow for efficient, decentralized maintenance of the cultural content directly by each partner country, an appropriate Extranet has to be developed. Finally, to facilitate sustainability of the project results, an appropriate organisational infrastructure has to be developed as well.

\subsection{Design of the MEDINA Cultural Content}

The design of the cultural content provided by MEDINA is founded on a set of general structuring concepts, comprising the notions of interesting spot, axis, thematic pathway and cultural framework. An interesting spot is any topic which is related to the cultural heritage of a specific country and can be of interest for a cultural tourist. An example for an interesting spot would be "The olive oil production in Tuscany". An axis defines a broad category of interesting spots, comprising, e.g., material heritage, performing art, folk tradition, handicraft or rituals (cf. Figure 1). A thematic pathway (pathway for short) defines a virtual itinerary about a cultural theme. It is composed of interesting spots, being part of potentially different cultural axes and includes a general introduction that presents the pathway's subject. Examples for pathways are "The pirates commerce" or "The carpets trade" (cf. Figure 1). A cultural framework, finally, is a collection of pathways concerning similar themes (e.g., "Markets courses" or "The myths culture" (cf. Figure 1). Mediterranean pathways and frameworks allow to highlight both, the common cultural background of Mediterranean countries and the individual contributions of the different civilizations in the Mediterranean basin. 


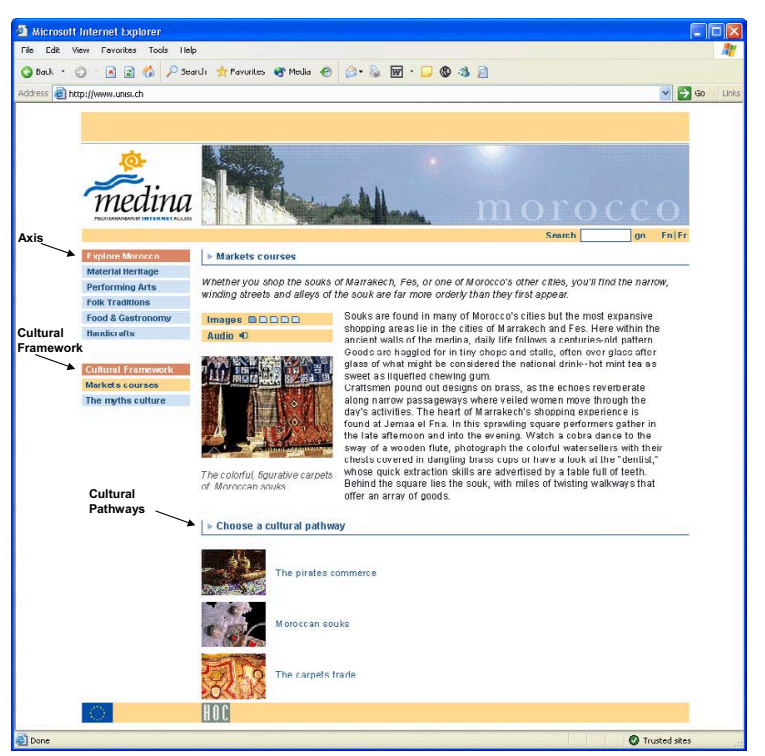

Figure 1. Exemplary MEDINA Web page

Finally, it has to be emphasised that axis, pathways and cultural frameworks are only one possible navigation structure for accessing interesting spots. Access is also provided through two, more traditional mechanisms, comprising geographical search and historical search.

\subsection{The MEDINA Technical Architecture}

The MEDINA technical architecture comprises a federation of national Web sites integrated through the MEDINA portal (cf. Figure 2).

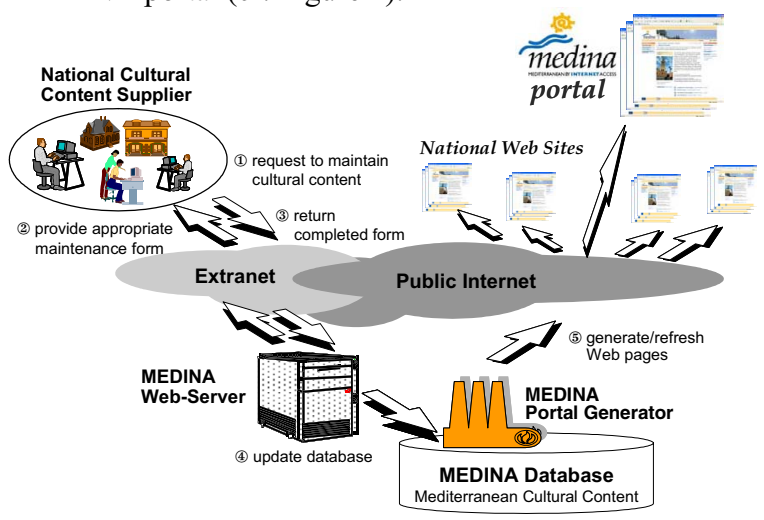

Figure 2. Overall MEDINA architecture

To facilitate integration and consistency among all national Websites and the MEDINA portal, all these systems share a common design schema. According to the W2000 design model [27], the schema is defined by a set of classes of information, a set of navigation structures, and a set of conceptual layout templates for the different types of Web pages. This design schema can be regarded as an application "skeleton", which captures the essential features common to all national Web sites and to the MEDINA portal. If a country has peculiar representation requirements, the design schema can be easily specialized and adapted to the national needs. A national Web site can be described as an instantiation of the common design schema or of its specialization. This design approach offers the prerequisite to build a portal generator which is responsible for the generation and update of Web pages, located either at the MEDINA portal or at the national Web sites. The portal generator dynamically updates contents and links within the corresponding Web pages, every time changes are made to the stored content by means of the Extranet (cf. below) [20], [22].

In addition to the portal generator, an Extranet is being developed, allowing partners to update the underlying database, storing cultural content in terms of textual and multimedia information, as well as linking information (cf. [19]). In order to ensure a homogeneous look and feel of cultural content published at the MEDINA portal, the already existing huge amount of content (in digital or non digital form) at the partner countries needs to be properly adapted before being entered into the Extranet. This means fragmenting, restructuring, and reshaping a piece of content to meet the communication goals of the project.

\section{Concepts for Realizing Ubiquity}

One way of enhancing the quality of access to cultural Web applications is, as already mentioned, to allow for ubiquity, thus providing device-independent, time-aware, location-aware and personalized services. This could assist cultural tourists not only in the course of the preparatory phase of a vacation by, e.g., automatically filtering cultural information according to a users preferences, but also during vacation itself, by delivering, e.g., location-dependent cultural information directly to the traveller's PDA (cf., e.g., [21]). In the following, we propose an architecture together with a set of concepts to realize ubiquitous cultural Web applications.

\subsection{The Customisation Architecture}

We consider the notion of customisation as being the basic concept for achieving ubiquity, in that a Web application is adapted towards its context [11]. The roots of customisation are manifold and can be found in user interfaces being either adaptive [7] or even intelligent and advisory [4], information filtering and recommender systems [16], adaptive hypertext and hypermedia [3] and mobile computing [1]. In ubiquitous Web applications, the classical user model employed for personalization purposes (cf., e.g., [14]) is generalized to a context model, adding primarily environmental data in terms of, e.g., location, time, device and network characteristics. 
To accomplish this basic idea, we employ a customisation architecture as depicted Figure 3. The context provides detailed information about the environment of a Web application and the Web application itself. Thereby the context influences not only the requirements as gathered by requirements elicitation but also triggers the actual customisation as soon as the context changes. A rule-based mechanism in terms of customisation rules is employed in order to specify the actual adaptations thereby influencing the MEDINA portal generator. Customisation rules are, in turn, determined by requirements.

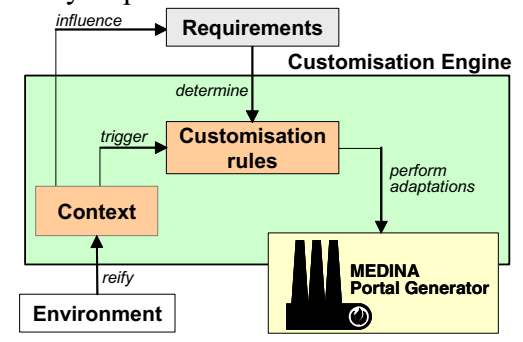

Figure 3. Overall Customisation Architecture

For a detailed description of the architecture the reader is referred to [5].

\subsection{Context}

The context comprises the circumstances of consumption relevant for a ubiquitous Web application in term of context properties, mainly dealing with the question "why to customise and when". For augmenting the MEDINA portal with ubiquity, we propose that at least the following context properties should be considered:

- Device: The device property refers to the demand for device-independence by identifying the device used, together with device profile information capturing the hardware and software capabilities in terms of, e.g., display size, memory, operating system, and kind of browser. Whereas the assistance of the MEDINA portal during initial travel planning will most likely be invoked using a PC, the actual on site visits during the travel will require some mobile devices like a PDA.

- User: Knowledge about the user takes into account the necessity of personalization. Cultural tourists are different to traditional tourist in that they are most often interested in very particular aspects of a countries' culture. Thus, amongst others, the preferences for a certain period or aspect of culture need to be taken into account in form of a profile.

- Network: In a mobile scenario the connection bandwidth is considerably different to wired connections and much more volatile. This is particularly the case when travelling in countries not yet fully covered with high bandwidth wireless networks. Consequently, the actually available bandwidth is a highly crucial factor in determining the transmittable information to the user.

- Location: Location copes with the need of mobile computing to provide location-aware services like e.g. navigation support. Technically the current location is determined by involving a positioning service like GPS or indoor tracking systems. Using a GSM smart phone allows to determine the actual GSM cell a user currently resides in. In terms of exploiting this location information, the cell identifier might not be sufficient but needs to be supplemented with additional location and geographic information to determine for example the road a tourist is moving along.

- Time: The context property time allows to customise the application with respect to certain timing constraints, e.g., opening hours of cultural sights or timetables of public transportation.

Since Web applications enforce the notion of sessions, these context properties need to be considered within the boundaries of sessions, i.e., each session has its own context. Furthermore, since the context within a session is subject to continuous changes, it is necessary to identify the most recent reification of the environment, which is further on called current context, using the latest timestamp. The current context comprises the current values of the context properties for a given interaction (e.g., the most recent) within the session of a ubiquitous Web application. Practice has shown that it is necessary to broaden the view on context in that a context should not only consider the current context at a given point in time but also historical information. This is necessary to be able to identify changes in the values of the context properties over time. Thus, context also needs to include a history dimension, in that a relevant context $\mathrm{C}$ can be formulated as a vector of context property values over time. For example, to determine user navigation patterns or the average throughput of a system, it is necessary to collect information about historic interactions. Summarizing, our understanding of an appropriate context model is shown in Figure 4 by means of a UML class diagram ${ }^{1}$. For more information about context models, it is referred to [12].

\subsection{Customisation Rules}

The adaptation of the MEDINA portal towards a certain context is specified in terms of customisation rules. For realising customisation rules, we propose the usage of the event/condition/action (ECA) mechanism which is well known in the area of active database

\footnotetext{
Please note that just those attributes and methods are shown in the context model which are used in the later customisation examples in Section 4 .
} 
systems [10]. The event part of the rule determines the events which are able to trigger the rule, thereby monitoring changes within the context model as well as explicit user requests. If the rule incorporates a condition, the condition is evaluated as soon as the rule is triggered. Whereas events specify when adaptation is potentially necessary, conditions determine whether (and which) adaptation is actually desired by considering context information. Conditions are in fact predicates which can be combined by means of logical operators. If the condition evaluates to true, the rule's action is executed.

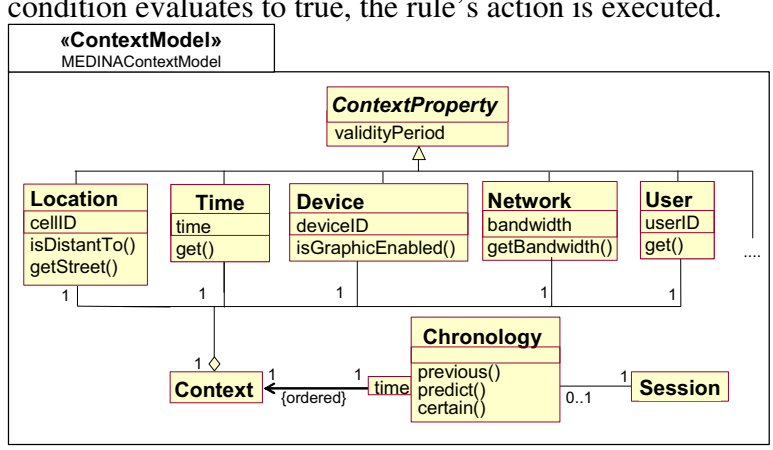

Figure 4. The Context Model

Actions are used to realise adaptations by augmenting the behaviour of the portal generator, e.g., selecting cultural information on bases of the user's current location.

\section{Ubiquity in MEDINA - A Usage Scenario}

This section applies some of the customisation concepts introduced in the previous section to the MEDINA portal by realising an ubiquitous cultural tourist guide for supporting the cultural tourist during vacation. On tour, a traveller will use a mobile device like a PDA or a smartphone to access the MEDINA portal. On such devices, not the full range of media formats like videos can be presented. Consequently, a text-only version of the requested cultural information should be presented when the tourist uses a non-graphic-enabled device. Since cultural sights are often located outside urban areas, connectability and bandwidth can vary considerably due to underdeveloped network coverage. Therefore, ubiquitous access requires that the cultural information is adapted to the available bandwidth to provide best quality of service. To enable the tourist to discover also cultural sights not laying on the mainstream tourist paths, navigation support should be given by the system. Route description and direction needs to be refreshed as the tourist travels along.

From these requirements, customisation rules are derived to customise the MEDINA portal towards ubiquity by taking into account the context and triggering the appropriate adaptations. Figure 5 shows part of the MEDINA common design schema (described in
Subsection 2.3) using $\mathrm{UML}^{2}$ with the customisation rules attached in UML annotations.

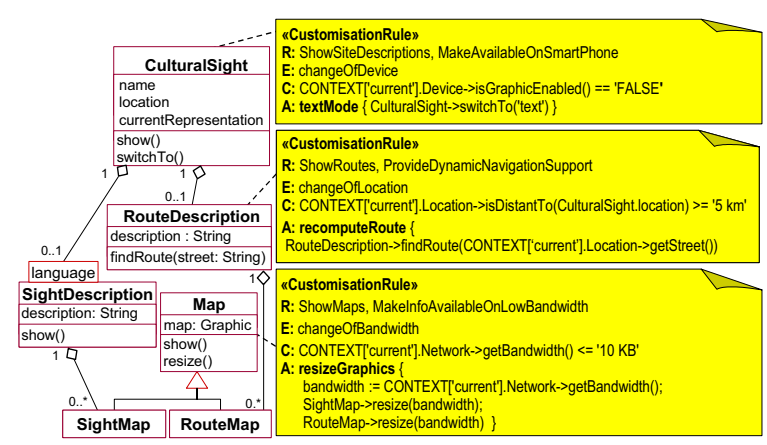

Figure 5. Examples of Customisation Rules ${ }^{3}$

These annotations are stereotyped with "CustomisationRule» to indicate their function. The specification of the requirement the customisation rules is realising is marked with " $\mathrm{R}:$ ". The " $\mathrm{E}:$ ", " $\mathrm{C}:$ ", and "A:" indicate the event, condition, and action of the customisation rule, respectively. The first rule specifies the requirement to use text only on non-graphic enabled devices. The event detects that the device changed, the condition evaluates the graphical capability of the device by accessing the device context and the action activates the adaptation method switchTo() of the customisable object Culturalsight. The second rule customises the graphic resolution according to the bandwidth. For this, the event detects bandwidth variations, the condition checks whether the bandwidth falls below $10 \mathrm{~KB}$, and the action resizes the two maps (SightMap and Routemap) proportionally. The last rule recalculates a route if the user's distance is more than $5 \mathrm{~km}$ away from those position which was used to last calculate the route to a cultural sight. The rule is triggered by the user's change of the location, the condition uses the method isDistantTo() to calculate the distance between the current position of the user and the position the route was calculated before. The action re-computes the new route taking the user's current position as input parameter.

\section{Conclusion}

The MEDINA portal described in this paper should facilitate the cultural tourist who lives a vacation as a learning experience. This offers an opportunity to discover unexploited resources of an area, including folk

\footnotetext{
2 Note that, although the common design schema of the MEDINA portal is represented using the W2000 modeling method which is a extension of UML, for simplicity reasons, we have used plain UML.

3 For readability reasons, we have introduced CONTEXT [<time $>$ ] as a shortcut for session.chronology. [<time>].context which would be the correct access path to context information according to Figure 4 .
} 
traditions, habits of the everyday life, rituals, history, or gastronomy. We have shown that the MEDINA portal could not only support the pre-planning of cultural vacations but could be also augmented to support the cultural tourists while travelling. In this way, two crucial issues of today's cultural Web applications are addressed, comprising quality of content and quality of access. Quality of content can be achieved by a Web portal approach combing a national as well as a Mediterranean perspective. Quality of access is provided by augmenting the MEDINA portal with ubiquity support. Three customisation examples are shown which demonstrate how to adapt the MEDINA portal to the tourists' context.

\section{References}

[1] J. Altmann, G. Leonhartsberger, M. Pichler, W. Schwinger, Th. Hofer, W. Retschitzegger, "Context-Awareness on Mobile Devices - the Hydrogen Approach", Proc. of the 36th Hawail Int. Conf. on System Sciences (HICSS), Hawaii, Jan., 2003.

[2] F., Bellas, "Standards for Second-Generation Portals", IEEE Internet Computing, 8(2), Mar./Apr., 2004.

[3] P. Brusilovsky, M. T. Maybury, "From adaptive hypermedia to adaptive Web", Communications of the ACM (CACM), 45(5), P. Brusilovsky/M. T. Maybury (eds.), 2002.

[4] J. M. Carroll, A. P. Aaronson, "Learning by Doing With Simulated Intelligent Help", Communications of the ACM (CACM), 31(9), Sept., 1988.

[5] A. Finkelstein, A. Savigni, G. Kappel, W. Retschitzegger, E. Kimmerstorfer, W. Schwinger, Th. Hofer, B. Pröll, Ch Feichtner, "Ubiquitous Web Application Development - A Framework for Understanding", Proc. of the 6th SCI Conference, Orlando, July, 2002.

[6] F. Garzotto, P. Paolini, M. Speroni, "Transnational portals for culture oriented tourism: the experience of the MEDINA project”, Int. Cult. Heritage Informatics Meeting, Paris, 2003.

[7] M. D. Good, J. A. Whiteside, D. R. Wixon, S. J. Jones, "Building a User-Derived Interface", Communications of the $A C M$ (CACM), 27(10), Oct., 1984.

[8] O'Grady, M.J., \& O'Hare, G.M.P., "Accessing Cultural Tourist Information via a Context Sensitive Tourist Guide", Information Technology and Tourism, 5(1), Cognizant Publishers, 2002.

[9] M. Haller, B. Pröll, W. Retschitzegger, A M. Tjoa, R. Wagner, "Integrating Heterogeneous Tourism Information in TIScover - The MIRO-Web Approach", Proc. of the Int. Conf. on Communication Technologies for Tourism (ENTER), Barcelona, Apr., 2000.

[10] G. Kappel, W. Retschitzegger, "The TriGS Active ObjectOriented Database System - An Overview", ACM SIGMOD Record, 27(3), Sept., 1998

[11] G. Kappel, B. Pröll, W. Retschitzegger, W. Schwinger, T. Hofer, "Modeling Ubiquitous Web Applications - The WUML Approach", Proc. of the Int. Workshop on Data Semantics in Web Information Systems, Yokohama, Nov., 2001.
[12] G. Kappel, W. Retschitzegger, E. Kimmerstorfer, W. Schwinger, Th. Hofer, B. Pröll, "Towards a Generic Customisation Model for Ubiquitous Web Applications", Proc. of the 2nd Int. Workshop on Web Oriented Software Technology (IWWOST), Spain, June, 2002.

[13] G. Kappel, B. Pröll, W. Retschitzegger, W. Schwinger, "Customisation for Ubiquitous Web Applications - A Comparison of Approaches", Int. Journal of Web Engineering and Technology (IJWET), 1(1), Inderscience Publishers 2003.

[14] A. Kobsa, "Generic User Modeling Systems", User Modeling and User-Adapted Interaction, 11(1-2), 2001.

[15] A. van Lamsweerde, Requirements Engineering in the Year 00: A Research Perspective, Proc. of the 22nd Int. Conf. on Software Engineering (ICSE), 2000.

[16] S. Loeb, D. Terry, "Information Filtering", Communications of the ACM (CACM), 35(12), Dec., 1992.

[17] T.W., Malone, et al., "Electronic markets and electronic hierarchies", Communications of the ACM, 30(6), 1987.

[18] Minerva Working Group5 (eds.), Handbook for quality in cultural Web sites, Version 1.2, Nov., 2003.

[19] B. Pröll, W. Retschitzegger, R.R. Wagner, "Extranet-based Maintenance and Customisation of Tourism Information", Proc. of the Euro Multimedia, Microprocessor Systems and Electronic Commerce (EMMSEC), IOS Press, France, Sept. 1998.

[20] B. Pröll, H. Sighart, W. Retschitzegger, H. Starck, "Ready for Prime Time - Pre-Generation of Web Pages in TIScover", Proc. of the Eighth Int. ACM Conf. on Information and Knowledge Management (CIKM), Kansas City, Nov., 1999.

[21] B. Pröll, W. Retschitzegger, "Discovering Next-Generation Tourism Information Systems - A Tour on TIScover", Journal of Travel Research, Sage Publications Inc., 39(2), Nov., 2000.

[22] M. Schrefl, M. Bernauer, E. Kapsammer, B. Pröll, W. Retschitzegger, T. Thalhammer, "Self-Maintaining Web Pages", Information Systems (IS), 28(8), Elsevier Science Ltd., 2003

[23] A. Schulz, "The Role of Global Computer Reservation Systems in the Travel Industry Today and in the Future", Newsletter Competence Center Electronic Markets, 6(2), 1996.

[24] A.G., Schuster, “A Delphi survey on electronic distribution channels for intermediaries in the tourism industry: The situation in German speaking countries, Buhalis, D. et al. (eds.), Proc. of the Int. Conf. on Information and Communication Technologies in Tourism (ENTER), Springer, 1998.

[25] Technical Annex of the MEDINA project, MEDINA Consortium, Apr., 2003.

[26] Travel Industry Assoc. of America, New Travel and Technology Report: 6,7 Million Adults Use the Internet For Travel Reservations, Jan., 1999.

[27] UWA Consortium, "The UWA Approach to Modeling Ubiquitous Web Applications", IST Mobile \& Wireless Telecommunications Summit, Thessaloniki, Greece, June, 2002.

[28] Ch. Wege, "Portal Server Technology", IEEE Internet Computing, 6(3), May/June, 2002.

[29] H. Werthner, S. Klein, "Information Technology and Tourism - A Challenging Relationship”, Springer, 1999. 